\title{
SOME CONSEQUENCES OF $(V=L)$ IN THE THEORY OF ANALYTIC SETS
}

\author{
R. W. HANSELL
}

\begin{abstract}
Following G. M. Reed's definition of a $Q$-set, we define a $Q_{\boldsymbol{A}}$-set to be any non- $\sigma$-discrete topological space with the property that each subset is (relatively) analytic (= Souslin-F set). Clearly every $\boldsymbol{Q}$-set is a $\boldsymbol{Q}_{\boldsymbol{A}}$-set. The discrete irrational extension of the space of real numbers is an example of a first countable hereditarily paracompact (Hausdorff) $Q_{\boldsymbol{A}}$-set which is not a $Q$-set.

THEOREM. $(V=L)$ Let $X$ be a first countable normal space all of whose subsets are analytic. Then $X$ is $\sigma$-discrete if and only if the product of $X$ with the space of irrational numbers is normal.

A new structural property of analytic sets is developed in order to utilize a proof technique due to Reed. Several corollaries are obtained on properties of completely additive-analytic families of sets in general metric spaces.
\end{abstract}

1. Introduction. A classical theorem of descriptive set theory states that if $X$ is an analytic subset of a Polish (complete separable metric) space, then $X$ is either countable or contains a homeomorph of the Cantor set (see e.g. [K, p. 479] or [Ha, p. 205]). Generalizations to nonseparable complete metric spaces have been subsequently obtained by Stone [ $\left.\mathbf{S}_{1}\right]$ (for the case when $X$ is a Borel set) and El'kin [E] ( $X$ again analytic), where the conclusion now states that $X$ is either $\sigma$-discrete ${ }^{1}$ or contains a homeomorph of the Cantor set. (Note that the $\sigma$-discrete subsets of a separable metric space are precisely the countable subsets.) There are absolute examples which show that these theorems are not true for analytic sets in arbitrary metric spaces (see [K, p. 514]).

There is, however, a corollary to the above theorems which raises an interesting problem when one removes the assumption of completeness. The corollary is the observation that if $X$ is an analytic subset of a complete (separable) metric space and every subset of $X$ is an analytic set, then $X$ is necessarily $\sigma$-discrete (respectively countable), since any homemorph of the Cantor set contains nonanalytic sets. In the present paper we will investigate the extent to which this result continues to hold without the assumption of completeness.

Reed $\left[\mathbf{R}_{\mathbf{2}}\right]$ defines a $Q$-set to be any topological space which is not $\sigma$-discrete but has the property that every subset is an $F_{\sigma}$-set of the space. Following this nomenclature, let us define a $Q_{A}$-set to be any space which is not $\sigma$-discrete but has the property that every subset is a relative analytic set. ${ }^{2}$

Received by the editors June 27, 1979 and, in revised form, October 8, 1979.

AMS (MOS) subject classifications (1970). Primary 54H05, 02K05, 04A15; Secondary 54E35, 54A25.

$K e y$ words and phrases. $(V=L)$, analytic set, $\sigma$-discrete, $Q$-set, $Q_{A}$-set, completely additive-analytic family, $\sigma$-discretely decomposable, d-family, metrizable space.

'A space is $\sigma$-discrete if it is a countable union of closed and discrete subsets.

${ }^{2}$ By an analytic subset of a topological space we mean a set which is the result of Souslin's operation-(A) applied to closed subsets of the space (see $\$ 2$ for a detailed definition). 
From the known results about $Q$-sets and analytic sets, we can easily deduce that the existence of a separable metric $Q_{A}$-set cannot be decided in the theory (ZFC) alone. First observe that if $k$ is an infinite cardinal and $k^{+}$denotes its successor, then the assumption $\left(2^{k}<2^{k^{+}}\right)$implies that any metric space of weight $<k$ and cardinality $>k$ cannot be a $Q_{A}$-set, since $X$ has at most $2^{k}$ analytic subsets $\left[\mathbf{S}_{2}\right.$, Theorem 23]. In particular, $\left(2^{\omega}<2^{\omega_{1}}\right)$ implies that no (uncountable) separable metric space is a $Q_{A}$-set. On the other hand, we have the well-known fact (due to Silver and Tall [T]) that under (MA $+\neg \mathbf{C H})$ every subset of the reals of cardinality $k$ is a $Q$-set (hence a $Q_{A}$-set) for $\omega_{1} \leqslant k<2^{\omega}$. (For a further discussion of $Q$-sets and their applications to problems in set-theoretic topology see [Ru], [ $\left.\mathbf{F}_{\mathbf{1}}\right]$, and $\left[\mathbf{R}_{\mathbf{1}}\right]$.)

Reed has shown that an analogous situation is also true for nonseparable metric $Q$-sets. Indeed, as announced in $\left[\mathbf{R}_{2}\right]$, it is even consistent with $(\mathbf{G C H})$ that there exist nonseparable metric $Q$-sets (hence again, nonseparable metric $Q_{A}$-sets). Previously, Reed had shown in $\left[\mathbf{R}_{\mathbf{1}}\right]$ that under Gödel's axiom of constructibility $(V=L)$, no first countable, normal, Hausdorff space can be a $Q$-set; in other words, such topological spaces which have the property that every subset is a relative $F_{\sigma}$-set must necessarily be a $\sigma$-discrete if $(V=L)$ holds. Our main objective in this paper is to show that under a slightly stronger normality assumption, this last result continues to hold for $Q_{A}$-sets. That this stronger normality condition is necessary is shown by the following (absolute) example.

EXAMPLE 1. There exists a first countable, Hausdorff and hereditarily paracompact space $X$ which is not $\sigma$-discrete but has the property that every subset is an $F_{\sigma \delta}$-set. We denote by $R, P$, and $Q$ the space of all real, irrational, and rational numbers respectively. Now let $X$ be the set of real numbers topologized so that a set is open in $X$ if and only if it has the form $U \cup K$, where $U$ is an open set in $R$ and $K$ is any subset of $P$. The space $X$ is known to be first countable, Hausdorff and hereditarily paracompact (see e.g. [En, Example 5.1.22] or [SS, Example 71]). The space $X$ is not $\sigma$-discrete, for otherwise $Q$ would be a $G_{\delta}$-set in $X$, and hence also in $R$ contrary to the Baire category theorem. To see that every subset of $X$ is an $F_{\sigma \delta}$-set, first observe that $P$ is $F_{\sigma \delta}$ in $X$ since every closed set in $R$ is closed in $X$. Now let $E$ be any subset of $X$. Since $P-E$ is open in $X, X-(P-E)=(E \cap P)$ $\cup Q$ is closed, and so the set

$$
[(E \cap P) \cup Q] \cap P=E \cap P
$$

is $F_{\sigma \delta}$ in $X$. Since the countable set $E \cap Q$ is clearly $F_{o \delta}$ in $X$, so is $E=(E \cap Q)$ $\cup(E \cap P)$. It follows that the space $X$ has all the requisite properties. ${ }^{3}$

A well-known property of the space $X$, originally due to Michael [M], is that the product space $X \times P$ is not normal. This must in fact be a property of any absolute example of a first countable normal $Q_{A}$-set in view of the following theorem:

\footnotetext{
${ }^{3}$ The reader will note that the space $X$ is not perfect, and so its Borel hierarchy is somewhat pathological. The first Corollary to our Theorem presented below shows that it is consistent with (ZFC) that no first countable perfectly normal space is a $Q_{A}$-set. On the other hand, in response to a question raised by the author, Professor Arnold Miller has shown in [Mi] that it is consistent with (ZFC) that there exists a separable metric $Q_{A}$-set which contains non-Borel subsets.
} 
THEOREM. $(V=L)$ Let $X$ be any normal space of character $<c$ with the property that each subset is relatively analytic. ${ }^{4}$ Then $X$ is $\sigma$-discrete if and only if the product space $X \times P$ is normal..$^{5}$

The proof of the Theorem will be given in the next section after some preliminary lemmas and definitions. Let us note here that the "only if" part of the conclusion holds without the assmption $(V=L)$ since any normal $\sigma$-discrete space $X$ is perfectly normal, and Morita [Mo] has shown that the product of $X$ with any metrizable space will then be perfectly normal.

From Morita's theorem we can also deduce immediately the following corollary to the above Theorem.

Corollary. $(V=L)$ Let $X$ be any first countable perfectly normal space (e.g., any metrizable space). Then $X$ is $\sigma$-discrete if and only if every subset of $X$ is relatively analytic.

We conclude this section with a second corollary to the above Theorem, the proof of which is almost verbatim that of the Corollary to Theorem 5 in $\left[\mathbf{R}_{\mathbf{1}}\right]$.

COROLlary. Let $X$ be a first countable space with the properties that each subset is analytic and the product space $X \times P$ is normal. Then it is independent of and consistent with (ZFC) that $X$ is metrizable.

Proof (cf. $\left.\left[\mathbf{R}_{1}\right]\right)$. Assume $(V=L)$. From $\left[\mathbf{F}_{2}\right]$ and the Theorem, $X$ is collectionwise Hausdorff and $\sigma$-discrete, and hence metrizable by a result of Reed [ $\left.\mathbf{R}_{\mathbf{3}}\right]$.

Assume (MA $+\neg \mathrm{CH}$ ). Then one can construct various kinds of first countable, perfectly normal Hausdorff $\sigma$-discrete spaces (in fact, normal Moore $\sigma$-discrete spaces) which are not metrizable (see [Ru, Chapter IV]).

2. Some lemmas on analytic sets and the proof of the Theorem. Let $\omega$ denote the set of natural numbers $\{1,2, \ldots\},{ }^{\omega} \omega$ the set of all sequences in $\omega$, and ${ }^{n} \omega$ the set of finite sequences of length $n \in \omega$. For $\phi \in{ }^{\omega} \omega, \phi \mid n$ will denote the element of ${ }^{n} \omega$ consisting of the first $n$ terms of $\phi: \phi \mid n=\left(\phi_{1}, \ldots, \phi_{n}\right)$. By a slight abuse of notation, we will sometimes denote members of the set ${ }^{n} \omega$ by $\phi \mid n$, without intending to specify a member of ${ }^{\omega} \omega$.

By an analytic set in a topological space $X$ we mean any subset $A$ of $X$ having the form

$$
A=\bigcup_{\phi} \bigcap_{n \in \omega} A_{\phi \mid n},
$$

where $\phi$ ranges over ${ }^{\omega} \omega$ and $A_{\phi \mid n}$ is a closed set in $X$ for each $\phi \mid n$ in ${ }^{n} \omega$ $(n=1,2, \ldots)$. We will always assume that the representation (1) has been

\footnotetext{
${ }^{4}$ Professor Heikki Junnila has noted that any space in which all singletons are analytic is necessarily a $T_{1}$-space.

${ }^{5}$ In my original statement and proof of this theorem the space $X$ was assumed to be first countable. The referee has observed that the proof actually shows that the space need only have character $<c$ (i.e., each point has a local base of cardinality $<c$ ). Reed's theorem (loc. cit.) can also be extended in the same way.
} 
"regularized" so that $A_{\phi \mid n+1} \subset A_{\phi \mid n}$ always holds (see [KMㄹ, XI, §5]).

If $\varphi \mid n$ and $\psi \mid n$ are members of ${ }^{n} \omega$, we write $\psi|n<\phi| n$ provided $\psi_{i}<\phi_{i}$ for $i=1, \ldots, n$, and we put

$$
T_{\phi \mid n}=\{\psi|n: \psi| n<\phi \mid n\}
$$

Similarly, if $\phi$ and $\psi$ are members of ${ }^{\omega} \omega$, we write $\psi<\phi$ provided $\psi|n<\phi| n$ for every $n \in \omega$, and we put

$$
T_{\phi}=\{\psi: \psi<\phi\} .
$$

The following lemma is proven in [BS, Lemma 4.6].

LEMMA 1. Let $A$ be as in (1). For any $\phi$ in ${ }^{\omega} \omega$ we have

$$
\bigcup_{\psi \in T_{\phi}} \bigcap_{n} A_{\psi \mid n}=\bigcap_{n} \bigcup_{\psi \in T_{\phi}} A_{\psi \mid n}=\bigcap_{n} \bigcup_{\psi \mid n \in T_{\phi \mid n}} A_{\psi \mid n} .
$$

The proof is an application of the pigeon-hole principle using the fact that the set $T_{\phi \mid n}$ is finite. We now deduce the following:

LEMMA 2. If $A$ is analytic in $X$, then $A$ has a representation of the type (1) satisfying the additional property that

$$
A_{\psi \mid n} \subset A_{\phi \mid n} \text { whenever } \psi|n<\phi| n .
$$

Proof. Choose closed sets $A_{\phi \mid n}$ in $X$ satisfying (1) and define

$$
A_{\phi \mid n}^{*}=\bigcup_{\psi \mid n \in T_{\phi \mid n}} A_{\psi \mid n}
$$

Each $A_{\phi \mid n}^{*}$ is closed in $X$ since $T_{\phi \mid n}$ is finite, and it is clear that the sets $A_{\phi \mid n}^{*}$ satisfy property (2). Let us show that

$$
A=\bigcup_{\phi} \bigcap_{n} A_{\phi \mid n}^{*}
$$

That the inclusion $\subset$ holds in (3) is immediate from the fact that $A_{\phi \mid n} \subset A_{\phi \mid n}^{*}$. On the other hand, Lemma 1 implies directly that

$$
\bigcap_{n} A_{\phi \mid n}^{*}=\bigcap_{n} \bigcup_{\psi \mid n \in T_{\phi \mid n}} A_{\psi \mid n}=\bigcup_{\psi \in T_{\phi}} \bigcap_{n} A_{\psi \mid n} \subset A,
$$

and (3) follows.

Finally, observe that $A_{\phi \mid n+1}^{*} \subset A_{\phi \mid n}^{*}$ follows immediately from the regularity of the sets $A_{\psi \mid n}$. That completes the proof.

As topological spaces, the set $\omega$ is given the discrete topo'ogy and ${ }^{\omega} \omega$ the product topology (and so is homeomorphic to the space of irrational numbers). We now give the proof of the main theorem; it closely parallels the proof of Reed's theorem cited in the introduction.

Proof (OF TheOREM). We first construct a Hausdorff space $Y$ of character $<c$ (i.e., each point has a local base of cardinality $<c$ ), and show that the properties of $X$ imply that $Y$ is normal. We then apply Fleissner's theorem $\left[F_{2}\right]$ to deduce that a certain discrete subset of $Y$ can be separated, and conclude from this that $X$ must be $\sigma$-discrete (cf. the proof of Theorem 5 in [ $\left.\mathbf{R}_{1}\right]$; also [Ru, p. 46]). 
Let ${ }^{<\omega} \omega$ denote the set of all (nonempty) finite sequences in $\omega$, and let

$$
Y=X \times\left({ }^{<\omega} \omega \cup\{\omega\}\right)
$$

We topologize $Y$ by specifying a local base for each point as follows:

(i) Each point of $X \times{ }^{<\omega} \omega$ is isolated.

(ii) Fix a point of the form $(x, \omega)$ and let $\left\{W_{r}(x): r \in R_{x}\right\}$ be a local base for the topology of $X$ at $x$, where the cardinality of the indexing set $R_{x}$ does not exceed $c$. Call a set $S \subset{ }^{<\omega} \omega$ a final segment if there is a $\phi \in{ }^{\omega} \omega$ such that for all $\psi \geqslant \phi$ there is $n(\psi, S)$ such that if $n \geqslant n(\psi, S)$, then $\psi \mid n \in S$. Then, for any final segment $S$ of $<\omega$ and map $g: S \rightarrow R_{x}$ the set

$$
W(S, g)=\{(x, \omega)\} \cup \bigcup_{\psi \mid n \in S} W_{g(\psi \mid n)}(x) \times\{\psi \mid n\}
$$

is defined to be a neighborhood of $(x, \omega)$.

It is easy to check that the final segments of ${ }^{<\omega} \omega$ are closed under finite intersections, and that the topology on $Y$ is well-defined and Hausdorff. Moreover, since there are at most $c$ maps from ${ }^{<\omega} \omega$ to $R_{x}$ for each $x$ in $X$, it follows that the character of $Y$ is $\leqslant c$.

We now proceed to show that $Y$ is normal. Clearly it suffices to show that whenever $H^{\prime}$ and $K^{\prime}$ are disjoint sets such that $H^{\prime} \cup K^{\prime}=X \times\{\omega\}$, then $H^{\prime}$ and $K^{\prime}$ can be separated by disjoint open sets of $Y$. To this end let

$$
H=\left\{x:(x, \omega) \in H^{\prime}\right\} \text { and } K=\left\{x:(x, \omega) \in K^{\prime}\right\} .
$$

Since $H$ and $K$ are analytic in $X$, there exist closed sets $H_{\phi \mid n}$ and $K_{\phi \mid n}$ in $X$, for each $\phi \mid n \in{ }^{<\omega} \omega$, satisfying properties (1) and (2) for $H$ and $K$ respectively.

Now the sets

$$
A=\bigcap_{n \in \omega} \bigcup_{\phi} H_{\phi \mid n} \times\{\phi\} \text { and } B=\bigcap_{n \in \omega} \bigcup_{\phi} K_{\phi \mid n} \times\{\phi\}
$$

are closed in the product space $X \times{ }^{\omega} \omega\left[\mathbf{K M}_{1}, \mathbf{X}, \S 4\right]$, and disjoint (since $(x, \phi) \in$ $A \cap B$ implies $x \in \cap_{n \in \omega} H_{\phi \mid n}$ and $x \in \bigcap_{n \in \omega} K_{\phi \mid n}$, but this implies that $x$ is in the empty intersection of $H$ and $K$ ). Hence, by normality, there exist disjoint open sets $U$ and $V$ in $X \times{ }^{\omega} \omega$ such that

$$
A \subset U \text { and } B \subset V \text {. }
$$

For each $\phi \mid n \in{ }^{<\omega} \omega$ let $W_{\phi \mid n}=\left\{\psi \in{ }^{\omega} \omega: \psi|n=\phi| n\right\}$. Then $W_{\phi \mid n+1} \subset W_{\phi \mid n}$ and $\left\{W_{\phi \mid n}\right\}_{n \in \omega}$ is a local base for $\phi$ in the space ${ }^{\omega} \omega$.

We now define for each $\phi \mid n$ open subsets of $X$ as follows:

$$
\begin{aligned}
& U_{\phi \mid n}=U\left\{W: W \text { is open in } X \text { and } W \times W_{\phi \mid n} \subset U\right\}, \\
& V_{\phi \mid n}=U\left\{W: W \text { is open in } X \text { and } W \times W_{\phi \mid n} \subset V\right\}
\end{aligned}
$$

It is immediate from the definitions that the sets in (8) are open in $X$ and satisfy

$$
U_{\phi \mid n} \cap V_{\phi \mid n}=\varnothing, \quad U_{\phi \mid n} \subset U_{\phi \mid n+1} \text { and } V_{\phi \mid n} \subset V_{\phi \mid n+1} \text {. }
$$


Finally, we define

$$
U^{\prime}=H^{\prime} \cup \bigcup_{\phi \mid n \in<\omega_{\omega}} U_{\phi \mid n} \times\{\phi \mid n\} \quad \text { and } \quad V^{\prime}=K^{\prime} \cup \bigcup_{\phi \mid n \in<\omega_{\omega}} V_{\phi \mid n} \times\{\phi \mid n\} \text {, }
$$

and proceed to show that these are open sets in $Y$ which separate $H^{\prime}$ and $K^{\prime}$. That $U^{\prime}$ and $V^{\prime}$ are disjoint follows immediately from (9) and (10), and the fact that $H^{\prime}$ and $K^{\prime}$ are disjoint.

To prove that $U^{\prime}$ is open in $Y$ let us first observe it suffices to show that to each $x \in H$ there corresponds a final segment $S$ of ${ }^{<\omega} \omega$ such that

$$
\psi \mid n \in S \quad \text { implies } x \in U_{\psi \mid n} \text {. }
$$

For then we can define $g(\psi \mid n)$ to be any index satisfying $W_{g(\psi \mid n)}(x) \subset U_{\psi \mid n}$ whenever $\psi \mid n \in S$, and it follows from (4) and (10) that $U^{\prime}$ contains the neighborhood $W(S, g)$ of $(x, \omega)$.

To prove (11), fix $x$ in $H$ and let $\phi$ be an element of ${ }^{\omega} \omega$ such that $x \in$ $\bigcap_{n \in \omega} H_{\phi \mid n}$. For any $\psi \geqslant \phi$, it follows from (2), (6) and (7) that $(x, \psi) \in A \subset U$. Hence, by (8), there exists some $n(\psi) \in \omega$ such that $x \in U_{\psi \mid n(\psi)}$, and consequently $x \in U_{\psi \mid n}$ for all $n \geqslant n(\psi)$ by (9). It follows that

$$
S=\{\psi \mid n: \psi \geqslant \phi \text { and } n \geqslant n(\psi)\}
$$

is a final segment satisfying (11). We conclude that $U^{\prime}$ and (by symmetry) $V^{\prime}$ are open in $Y$, proving $Y$ is normal.

By Fleissner's theorem $\left[\mathbf{F}_{2}\right], Y$ is collectionwise Hausdorff. Consequently, for each $x$ in $X$ there is an open set $G_{x}$ in $Y$ such that $(x, \omega)$ belongs to $G_{x}$ and the members of the collection $\left\{G_{x}\right\}_{x \in X}$ are pairwise disjoint. By (4) we can choose, for each $x$ in $X$, elements $r_{x}$ in $R_{x}, n_{x}$ in $\omega$, and $\psi_{x}$ in ${ }^{\omega} \omega$ such that

$$
W_{r_{x}}(x) \times\left\{\psi_{x} \mid n_{x}\right\} \subset G_{x} .
$$

For each $\psi \mid n$ in ${ }^{<\omega} \omega$ we now define

$$
D_{\psi \mid n}=\left\{x \in X: \psi_{x}\left|n_{x}=\psi\right| n\right\} .
$$

Then one easily checks that each of the sets $D_{\psi \mid n}$ is closed and discrete in $X$, and that $X=\cup_{\psi \mid n \in<\omega_{\omega}} D_{\psi \mid n}$. Since the set ${ }^{<\omega} \omega$ is countable, $X$ is $\sigma$-discrete, and that completes the proof of the Theorem.

In the next section we give some applications to the study of completely additive-analytic families.

3. Completely additive-analytic families. The property of a space having all subsets analytic can be extended to families of sets in the following way. A family $Q$ of subsets of a space $X$ is said to be completely additive-analytic (or caa) in $X$ if for every subfamily $\mathcal{Q}^{\prime}$ of $\mathcal{Q}$, the union $\cup Q^{\prime}$ is analytic in $X$. Completely additive-Borel families are defined similarly. In $\left[\mathbf{H}_{1}\right]$ we showed that a caa family of pairwise disjoint sets in a complete metric space is $\sigma$-discretely decomposable ( $\sigma \mathrm{dd}){ }^{6}$ This was the basis for extending a substantial part of the classical theory of

\footnotetext{
${ }^{6} \mathrm{~A}$ family $\mathcal{E}$ of subsets of a space $X$ is $\sigma$-discretely decomposable if there exist sets $E_{n}$, for each $E$ in $\mathcal{E}$ and $n$ in $\omega$, such that $E=\bigcup_{n \in \omega} E_{n}$ and $\left\{E_{n}: E \in \mathcal{E}\right\}$ is discrete in $X$ for fixed $n$.
} 
Borel measurable mappings to nonseparable metric spaces (see $\left[\mathbf{H}_{1}\right]$ and $\left[\mathbf{H}_{2}\right]$ ). Kaniewski and Pol in [KP] extended this result to include the case of point-finite families.

Recently in $\left[\mathbf{F}_{3}\right]$ Fleissner treated the Kaniewski-Pol result without the assumption of completeness as an axiom which he calls Proposition P. Fleissner shows that if there is a model of set theory with a supercompact cardinal, then there is a model in which Proposition $P$ is valid. His proof reveals an interesting relationship between $\sigma \mathrm{dd}$ families, caa families, and d-families, ${ }^{7}$ and Fleissner asks if there is any evidence to affirm that these results are implied by $(V=L)$. The main theorem of this paper together with the two corollaries to follow would appear to support such an affirmation.

Corollary 1. $(V=L)$ A caa family of pairwise disjont subsets of any metric space is a d-family.

Proof. Let $\mathcal{Q}=\left\{A_{t}: t \in T\right\}$ be such a family of nonempty sets relative to the metric space $\cup \mathbb{Q}$. let $x_{t} \in A_{t}$ be arbitrarily chosen for each $t \in T$, and put $X=\left\{x_{t}: t \in T\right\}$. Then for all $S \subset T$ we have

$$
\left\{x_{t}: t \in S\right\}=X \cap \bigcup_{t \in S} A_{t},
$$

showing that every subset of $X$ is relatively analytic. It follows from the Theorem that $X$ is $\sigma$-discrete, and hence that $A$ is a d-family.

REMARKs. The above corollary is, of course, true for any first countable $T_{1}$-space $X$ such that $X \times P$ is normal. I do not know if it remains true (even for metric spaces) if "pairwise disjoint" is replaced by "point-finite". The best I can do in this case is to point out that every selector will have a $\sigma$-discrete subset of the same cardinality by Lázár's theorem ([J, Appendix 3]; cf. also the proof of Theorem 1 in [KP]). Also, let us point out that Corollary 1 is emphatically not true for pointcountable families, even for completely additive- $F_{\sigma}$ families in the space of real numbers, as the following example shows.

Example 2. Use the Hausdorff $\boldsymbol{\aleph}_{1}$-sum Theorem [K, p. 484] to get $R=$ $\cup_{\alpha<\omega_{1}} G_{\alpha}$ where, for each $\alpha, G_{\alpha}$ is a $G_{\delta}$-set in $R, G_{\alpha} \subset G_{\alpha+1}$, and there exists some $x_{\alpha} \in G_{\alpha+1}-G_{\alpha}$. Then one easily checks that the family $\left\{R-G_{\alpha}: \alpha<\omega_{1}\right\}$ is point-countable, completely additive- $F_{\sigma}$ in $R$ and has the uncountable (hence non- $\sigma$-discrete) set $\left\{x_{\alpha}: \alpha<\omega_{1}\right\}$ as a selector.

If one assumes $(\mathrm{CH})$, then there also exists an example which is completely additive- $G_{\delta}$ (see [KP, Example 1]).

Consider now the following concepts introduced by Pol in [P]. Fix a family $\mathbb{Q}$ of subsets of $X$. A subset $E$ of $X$ is said to be $Q$-discrete provided we can choose a member $A_{x}$ of $Q$, for each $x \in E$, so that $A_{x} \cap E=\{x\}$. If $X$ is a topological space, $\mathbb{Q}$ is said to be weakly discrete provided that every $\mathbb{Q}$-discrete set is o-discrete. (Note that every d-family is weakly discrete and that the two notions coincide for disjoint families.)

\footnotetext{
${ }^{7} \mathrm{~A}$ family $\boldsymbol{E}$ of subsets of a space $X$ is a $d$-family if every selector for $\mathcal{E}$ is $\sigma$-discrete; i.e., if $S=\left\{x_{E} \in E: E \in \mathcal{E}\right\}$, then $S$ is $\sigma$-discrete.
} 
In [P] Pol proves an interesting theorem "On $\sigma$-discrete reduction" showing that every weakly discrete point-countable covering of a metric space $X$ consisting of sets of weight $\leqslant \aleph_{1}$ has a $\sigma$-discrete refinement. Examples are also given to show that neither the point-countability nor the weight restriction can be omitted in general. It is also shown that every completely additive-extended-Borel family (in the sense of $\left.\left[\mathbf{H}_{3}\right]\right)$ in a complete metric space is weakly discrete.

In $\left[\mathbf{H}_{4}\right]$ we use a result of Martin and Solovay [MS] to show that it is consistent with (MA $+\neg \mathbf{C H}$ ) that there exists a point-countable completely additive-analytic family of subsets of the reals which is not weakly discrete. We can now deduce as a corollary to our Theorem that it is consistent for all such families (in any metric space) to be weakly discrete.

COROllary 2. $(V=L)$ Every caa family $\mathbb{Q}$ of subsets of a metric space is weakly discrete. If, in addition, $\mathcal{Q}$ is point-countable and consists of sets of weight $\leqslant \aleph_{1}$, then $Q$ has a $\sigma$-discrete refinement.

Proof. Suppose $E$ is $\mathbb{Q}$-discrete, and, for each $x \in E, A_{x}$ is a member of $\mathcal{Q}$ such that $A_{x} \cap E=\{x\}$. If $M$ is any subset of $E$, then clearly $M=E \cap \cup_{x \in M} A_{x}$, and so $M$ is analytic in $E$. Hence, by our theorem, $E$ is $\sigma$-discrete as required. The second statement follows immediately from the theorem of Pol's cited above.

We conclude by listing several questions which remain open.

Question 1. Does $(V=L)$ imply that every point-finite caa family of subsets of a metric space is a d-family? In fact, are such $\sigma \mathrm{dd}$ ? (Cf. $\left[\mathbf{F}_{3}\right]$.)

Question 2. Does $(V=L)$ imply that every point-countable caa family of subsets of a metric space has a $\sigma$-discrete refinement?

Question 3. Is every point-countable, caa, d-family of subsets of a complete metric space $\sigma \mathrm{dd}$ ?

\section{BIBLIOGRAPHY}

[BS] D. W. Bressler and M. Sion, The current theory of analytic sets, Canad. J. Math. 16 (1964), 207-230.

[E] A. G. El'kin, A-sets in complete metric spaces, Soviet Math Dokl. 8 (1967), 874-877.

[En] R. Engelking, General topology, PWN, Warsaw, 1976.

[F] W. G. Fleissner, Current research on $Q$-sets, Proc. Bolyai Janos Conf. on Topology, Budapest, 1978.

$\left[\mathbf{F}_{2}\right]$ , Normal Moore spaces in the constructible universe, Proc. Amer. Math. Soc. 46 (1974), 294-298.

[F $\left.\mathbf{F}_{3}\right] \ldots$, An axiom for nonseparable Borel theory, Trans. Amer. Math. Soc. 251 (1979), 309-328.

[H] R. W. Hansell, Borel measurable mappings for nonseparable metric spaces, Trans. Amer. Math. Soc. 161 (1971), 145-169.

$\left[\mathrm{H}_{2}\right] \ldots$, On Borel mappings and Baire functions, Trans. Amer. Math. Soc. 194 (1974), 195-211.

$\left[\mathrm{H}_{3}\right] \ldots$, On the nonseparable theory of $k$-Borel and $k$-Soulsin sets, General Topology and Appl. 3 (1973), 161-195.

[H4 _ A note on complete additive-analytic families, (in preparation).

[Ha] F. Hausdorff, Mengenlehre, De Gruyter, Berlin, 1937; English transl., Chelsea, New York, 1957.

[J] I. Juhasz, Cardinal functions in topology, North-Holland, Amsterdam, 1971.

[KP] J. Kaniewski and R. Pol, Borel-measurable selectors for compact-valued mapping in the nonseparable case, Bull. Acad. Polon. Sci. 23 (1975), 1043-1050.

[K] K. Kuratowski, Topologie, Vol. 1, PWN, Warsaw, 1958; English transl., Academic Press, New York, 1966. 
[KM, K. Kuratowski and A. Mostowski, Set theory, Notrh-Holland, Amsterdam, 1967.

$\left[\mathbf{K M}_{2}\right] \ldots$, Set theory (with an introduction to descriptive set theory), North- Holland, Amsterdam, 1976.

[MS] D. A. Martin and R. M. Solovay, Internal Cohen extensions, Ann. Math. Logic 2 (1970), 143-178.

[M] E. Michael, The product of a normal space and a metric space need not be normal, Bull. Amer. Math. Soc. 69 (1963), 375-376.

[Mi] A. Miller, Generic Souslin sets, Abstracts Amer. Math. Soc. 1 (1980), p. 6. Abstract \#773-04-4.

[Mo] K. Morita, On the product of a normal space with a metric space, Proc. Japan Acad. 39 (1963), 148-150.

[P] R. Pol, Note on decompositions of metrizable spaces. II, Fund. Math. 100 (1978), 129-143.

$\left[\mathbf{R}_{1}\right]$ G. M. Reed, On normality and countable paracompactness, Fund. Math. (to appear).

$\left[\mathbf{R}_{2}\right] \ldots, Q$-sets and Shelah's principle, Notices Amer. Math. Soc. 26 (1979), A-124.

$\left[\mathbf{R}_{3}\right] \ldots$, Concerning first countable spaces, Fund. Math. 74 (1972), 161-169.

[Ru] M. E. Rudin, Lectures on set-theoretic topology, CBMS Regional Conf. Ser. Math., no. 23, Amer. Math. Soc., Providence, R.I., 1975.

[SS] L. A. Steen and J. A. Seebach, Jr., Counterexamples in topology, 2nd ed., Holt, Rinehart, and Winston, New York, 1978.

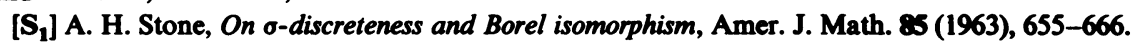

$\left[\mathrm{S}_{2}\right]$, Non-separable Borel sets, Dissertationes Math. (Rozprawy Mat) 28 (1962).

[T] F. D. Tall, Set-theoretic consistency results and topological theorems concerning the normal Moore space conjecture and related problems, Disserationes Math. (Rozprawy Mat.) 148 (1977).

Department of Mathematics, University of Connecticut, Storrs, Conbcticut 06268 